\title{
A retrospective study comparing the length of admission of medium secure unit patients admitted in the three decades since 1985
}

\author{
Charles H. Earnshaw, ${ }^{1}$ Lucy Shaw, ${ }^{2}$ Deepu Thomas, ${ }^{3}$ Owen Haeney $^{4,5}$
}

BJPsych Bulletin (2019) 43, 154-157, doi:10.1192/bjb.2018.88

\begin{abstract}
${ }^{1}$ Salford Royal NHS Foundation Trust, UK; ${ }^{2}$ Greater Manchester Mental Health NHS Foundation Trust, UK; ${ }^{3}$ Priory Healthcare, Kemple View Hospital, Blackburn, UK; ${ }^{4}$ Forensic Mental Health Service, South Australia; ${ }^{5}$ Discipline of Psychiatry, The University of Adelaide, Australia

Correspondence to Dr Charles Earnshaw (charles.earnshaw@cantab.net) First received 15 Mar 2018, final revision 30 Aug 2018, accepted 25 Sep 2018 (C) The Authors 2018. This is an Open Access article, distributed under the terms of the Creative Commons Attribution-NonCommercialNoDerivatives licence (http:// creativecommons.org/licenses/by-ncnd/4.0/), which permits noncommercial re-use, distribution, and reproduction in any medium, provided the original work is unaltered and is properly cited. The written permission of Cambridge University Press must be obtained for commercial re-use or in order to create a derivative work.
\end{abstract}

Aims and method Admissions of patients to secure forensic hospitals are often lengthy. Previous research has examined factors associated with prolonged admission, but studies analysing admission data at a single medium secure unit (MSU) over a prolonged time period are lacking. We compared admission data for all patients admitted to a MSU in England during the years 1985, 1995, 2005 and 2012.

Results The median length of admission increased from 167 days in 1985 to 580 days in 2012, though not in the intervening cohorts. There have been changes in the discharge destination of patients, away from independent accommodation in the community towards further care or supported accommodation.

Clinical implications The results suggest a change in the delivery of care. Further studies should be performed to assess whether the same trends exist at other sites. If these trends are also found elsewhere, this should trigger a specialty-wide discussion about admission length and its effects on bed availability.

Declaration of interest None.

Keywords Forensic mental health services; forensic psychiatry; length of stay.
Forensic hospitals, including high, medium and low secure services, have important roles in the treatment of psychiatric patients with a criminal history. Patients are admitted to these specialised services when it is felt they pose a risk to others, often due to a history of serious violence or other offending behaviours. The care of these patients, now more than ever, involves a multidisciplinary approach. ${ }^{1,2}$

There is an expanding body of literature analysing the outcomes of admission to medium secure units (MSUs). These studies suggest that patients are at significant risk of readmission and, sadly, at significantly increased risk of death compared with the general population. ${ }^{3-6}$ Admissions to MSUs are now often lengthy, ${ }^{7-9}$ findings that were echoed by a recent study analysing length of stay data in high secure units across Europe. ${ }^{10}$ Factors identified as associated with a longer length of stay include a diagnosis of a psychotic disorder, detention under a restriction order in particular, those under section $37 / 41$ (Section 37 is a court-issued order that means the patient will be sent to hospital rather than prison, and Section 41 is a so-called 'restriction order' that is designed to reduce the risk to the general public) of the Mental Health Act 1983 (amended
2007) - poor treatment response and the seriousness of the index offence. ${ }^{8}$ However, data from single sites over a prolonged period of time are lacking.

The objective of our study was to observe trends in the diagnoses, length of stay and discharge of patients admitted to a local MSU in the years 1985, 1995, 2005 and 2012. We also considered what factors or changes in service provision over the study period had affected the length of admission and discharge locations of our medium secure service.

\section{Materials and method}

Ethical approval for this study was gained from the local clinical audit department as a service evaluation and did not require approval from the local Research Ethics Committee. Non-anonymised data required by the study were gathered by one author (D.T.), and anonymised data were subsequently analysed by the remaining authors.

All male and female patients admitted to the MSU during the years 1985, 1995, 2005 and 2012 were included in the study. The admission year of 2012 was chosen (rather 


\begin{tabular}{|c|c|c|c|c|}
\hline Diagnosis & 1985 & 1995 & 2005 & 2012 \\
\hline Paranoid schizophrenia & $32(67 \%)$ & $60(92 \%)$ & $26(70 \%)$ & $24(80 \%)$ \\
\hline Schizoaffective disorder & $1(2 \%)$ & $0(0 \%)$ & $5(14 \%)$ & $3(10 \%)$ \\
\hline Mood disorder, manic episode & $0(0 \%)$ & $0(0 \%)$ & $0(0 \%)$ & $1(3 \%)$ \\
\hline Bipolar disorder & $7(15 \%)$ & $2(3 \%)$ & $2(5 \%)$ & $0(0 \%)$ \\
\hline Depressive episode & $0(0 \%)$ & $1(2 \%)$ & $1(3 \%)$ & $1(3 \%)$ \\
\hline Recurrent depressive disorder & $0(0 \%)$ & $0(0 \%)$ & $1(3 \%)$ & $0(0 \%)$ \\
\hline Affective mood disorder & $0(0 \%)$ & $0(0 \%)$ & $0(0 \%)$ & $1(3 \%)$ \\
\hline Antisocial personality disorder & $2(4 \%)$ & $0(0 \%)$ & $0(0 \%)$ & $0(0 \%)$ \\
\hline Emotionally unstable personality disorder & $0(0 \%)$ & $0(0 \%)$ & $1(3 \%)$ & $0(0 \%)$ \\
\hline Organic & $1(2 \%)$ & $1(2 \%)$ & $0(0 \%)$ & $0(0 \%)$ \\
\hline Undetermined & $5(10 \%)$ & $1(2 \%)$ & $1(3 \%)$ & $0(0 \%)$ \\
\hline
\end{tabular}

\begin{tabular}{|c|c|c|c|c|}
\hline & \multicolumn{4}{|c|}{ Year } \\
\hline & 1985 & 1995 & 2005 & 2012 \\
\hline Median duration of admission, days \pm s.d. & $167 \pm 299$ & $114 \pm 425$ & $110 \pm 566$ & $580 \pm 453$ \\
\hline Minimum duration, days & 1 & 1 & 3 & 3 \\
\hline Maximum duration, days & 1662 & 1952 & 2297 & $\overline{\text { Unknown }^{a}}$ \\
\hline
\end{tabular}

a. The maximum duration is unknown for this cohort owing to ongoing admission.

than a later year) as it provided sufficient time from admission to the date of data collection for treatment courses and possible discharges to be assessed.

One hundred and seventy-nine patient records were included in this study. The date of data collection was 4 April 2016. No patients were excluded from the study. Electronic records were analysed for a variety of criteria, including age on admission, date of admission, date of discharge, diagnosis, source of admission, location of discharge and convictions on admission. In the 2012 cohort, four patients had not yet moved on from the MSU on the date of data collection. Their discharge date was recorded as the date of data collection to give a minimum median length of stay for the 2012 cohort. These patients were excluded from the analysis of discharge destination.

Data compilation and analysis were performed in Microsoft Excel (Microsoft Corporation, Redmond, WA, USA). Statistical analysis comparing length of admission of different cohorts was performed with one-way ANOVA using an internet-based calculator (http://www.statisticslectures.com/calculators).

\section{Results}

There were 47, 65, 37 and 30 patients admitted to the MSU in 1985, 1995, 2005 and 2012, respectively. The majority of these patients were diagnosed with paranoid schizophrenia. The primary diagnoses of patients included in the different cohorts are shown in Table 1. The average age of the patient population varied little over the time period included in our study.

The median length of admission increased dramatically in our final cohort, from 167 days in 1985 to 580 days in 2012 (Table 2). According to a one-way ANOVA test, the median durations of the first and last cohorts, but not the intervening cohorts, differed significantly from one another $(P<0.01)$.

The discharge location also showed changes over the study period (Table 3). Fewer patients were discharged directly to their home (54\% in the 1985 cohort and $13 \%$ in the 2012 cohort), and more patients were discharged to other forms of psychiatric hospital, such as other MSUs (0\% in $1985,3 \%$ in 1995 and $2005,17 \%$ in 2012) or to low secure units (10\% in the 1985 cohort compared with $33 \%$ in the 2012 cohort).

\section{Discussion}

The main finding of this study is that the duration of admission has increased significantly in the three decades since the initial cohort, with patients now remaining for a median of close to two years in the MSU. This correlates with a reduction in the number of new admissions per year, down from a peak of 65 in 1995 to 30 in 2012. The duration of admission appears to have been relatively stable in the decades prior to the 2012 cohort; only in this cohort did the length of admission increase significantly. 


\begin{tabular}{|c|c|c|c|c|}
\hline Discharge location & 1985 & 1995 & 2005 & 2012 \\
\hline Police custody & $0(0 \%)$ & $0(0 \%)$ & $0(0 \%)$ & $1(3 \%)$ \\
\hline Prison & $5(10 \%)$ & $7(11 \%)$ & $6(16 \%)$ & $3(10 \%)$ \\
\hline Low secure psychiatric hospital & $5(10 \%)$ & $4(6 \%)$ & $8(22 \%)$ & $10(33 \%)$ \\
\hline Other MSU & $0(0 \%)$ & $2(3 \%)$ & $1(3 \%)$ & $5(17 \%)$ \\
\hline High secure psychiatric hospital & $4(8 \%)$ & $5(8 \%)$ & $1(3 \%)$ & $1(3 \%)$ \\
\hline Remained in our MSU & $0(0 \%)$ & $0(0 \%)$ & $0(0 \%)$ & $4(13 \%)$ \\
\hline Supported accommodation & $8(17 \%)$ & $28(43 \%)$ & $10(27 \%)$ & $2(7 \%)$ \\
\hline Home & $26(54 \%)$ & $17(26 \%)$ & $9(24 \%)$ & $4(13 \%)$ \\
\hline Died & $0(0 \%)$ & $0(0 \%)$ & $2(5 \%)$ & $0(0 \%)$ \\
\hline No information & $0(0 \%)$ & $2(3 \%)$ & $0(0 \%)$ & $0(0 \%)$ \\
\hline
\end{tabular}

The four patients in the 2012 cohort still in the MSU on 1 April 2016 each had a length of stay of at least three years and three months. Their final length of admission may be significantly longer, and we cannot know by how much the median length of stay is an underestimate.

Recommendations regarding treatment of mentally disordered offenders were made in the Glancy and Butler reports. ${ }^{11,12}$ These reports informed the development of the regional secure units (now known as MSUs) to complement the existing special hospitals (now known as high secure hospitals). An upper limit for length of stay of two years in the regional secure units was suggested, but this is now regularly exceeded, as this study shows. The increasing length of stay in MSU has been criticised, being deemed 'too long in very expensive and often unsuitable provision' in a report by the Schizophrenia Commission. ${ }^{13}$

Despite the aforementioned criticism of the increasing length of stay and the undoubted expense of a medium secure bed, these services have the potential to save society a significant financial burden. One report suggests an average saving of over $£ 600000$ per patient transferred from prison to psychiatric units.

Information regarding the length of stay at a single site has been investigated previously. ${ }^{14-17}$ However, no studies have investigated how the length of stay has changed over a prolonged period of time. Therefore, we feel that the data provided by our study add to the literature and provide a primary example of how length of admission has changed across a significant period of time. When comparisons were made with these early studies, the length of admission was comparable with that of the earliest cohort of our study. For example, in one paper published in 1981, the vast majority of patients were discharged in less than one year, which fits with the length of stay of the 1985 cohort in our study. ${ }^{14}$ It would be interesting to see modern studies in these other hospitals, to identify whether they have witnessed similar increases in length of stay.

One of the major changes since 1985 is in how patients are treated. In the older cohorts, the principal role of the forensic mental health service was to ensure that the symptoms of the patient's mental illness had reduced or resolved; offending risk related to other factors such as personality, substance misuse, social circumstances or life choices was often not felt to be the domain of mental health services. This underwent a significant change in the following decades. Mental health services now provide far broader care to address these other aspects, as evidenced by the essential roles of the multidisciplinary team ${ }^{1,2}$ and the adoption of recovery principles. These important changes are time and labour intensive, and as such may be a contributing factor to the increased length of stay.

In the analysis of the discharge locations and admission sources of these patient cohorts, certain patterns emerged. Far fewer patients are discharged directly to their home. Our patients are often discharged to long-term MSUs, lower security psychiatric units or supported accommodation. Notably, therefore, despite the increasing length of stay, fewer patients are discharged directly into independent accommodation in the community. Numerous studies have provided detailed analysis of the follow-up of patients discharged from forensic psychiatry units. ${ }^{3-6}$ Given the risks inherent in these patients returning to day-to-day life, further care in supported environments may reduce risk to others at a population level. Responses to serious untoward incidents have changed over time ${ }^{18,19}$ and may now be more likely to lead to greater restrictions for patients. This in turn may contribute to the increasing lengths of stay described above.

There are limitations to our study. As our cohort was from a single MSU, the general applicability of our findings may be limited. It is recommended that further research be undertaken to examine whether the trends identified here are reproduced in other MSUs.

\section{Funding}

C.H.E. is funded by a National Institute for Health Research Academic Clinical Fellowship.

\section{About the authors}

Charles H. Earnshaw $\odot$ is an academic clinical fellow at Salford Royal NHS Foundation Trust, UK; Lucy Shaw is a forensic psychiatry registrar at Greater Manchester Mental Health NHS Foundation Trust, Prestwich, UK; Deepu Thomas is a consultant forensic psychiatrist at Priory Healthcare, Kemple View Hospital, Blackburn, UK; and Owen Haeney is a consultant forensic psychiatrist at the Forensic Mental Health Service, James Nash House, 
Oakden and a clinical lecturer in the Discipline of Psychiatry at the University of Adelaide, Australia.

\section{References}

1 Clarke C, Lumbard D, Sambrook S, Kerr K. What does recovery mean to a forensic mental health patient? A systematic review and narrative synthesis of the qualitative literature. J Forensic Psychiatry Psychol 2016; 27 38-54.

2 Doyle M, Logan C, Ludlow A, Holloway J. Milestones to recovery: preliminary validation of a framework to promote recovery and map progress through the medium secure inpatient pathway. Crim Behav Ment Health 2012; 22(1): 53-64.

3 Davies S, Clarke M, Hollin C, Duggan C. Long-term outcomes after discharge from medium secure care: a cause for concern. Br J Psychiatry 2007; 191: 70-4.

4 Blattner R, Dolan M. Outcome of high security patients admitted to a medium secure unit: the Edenfield Centre study. Med Sci Law 2009; 49(4): 247-56.

5 Maden A, Rutter S, McClintock T, Friendship C, Gunn J. Outcome of admission to a medium secure psychiatric unit. Short- and long-term outcomes. Br J Psychiatry 1999; 175: 313-6.

6 Falla S, Sugarman P, Roberts L. Reconviction after discharge from a regional secure unit. Med Sci Law 2000; 40(2): 156-7.

7 Sharma A, Dunn W, O'Toole C, Kennedy HG. The virtual institution: cross-sectional length of stay in general adult and forensic psychiatry beds. Int J Ment Health Syst 2015; 30(9): 25.

8 Shah A, Waldron G, Boast N, Coid JW, Ullrich S. Factors associated with length of admission at a medium secure forensic psychiatric unit. J Forensic Psychiatry Psychol 2011; 22(4): 496-512.
9 Davoren M, Byrne $\mathrm{O}, \mathrm{O}^{\prime}$ Connell $\mathrm{P}, \mathrm{O}$ 'Neill H, O'Reilly K, Kennedy HG. Factors affecting length of stay in forensic hospital setting: need for therapeutic security and course of admission. BMC Psychiatry 2001; 15: 301.

10 Sampson S, Edworthy R, Völlm B, Bulten E. Long-term forensic mental health services: an exploratory comparison of 18 European countries. Int J Forensic Mental Health 2016; 15(4): 333-51.

11 Glancy Committee. Revised Report of the Working Party on Security in NHS Psychiatric Hospitals. HMSO, 1974.

12 Butler Committee. Report of the Committee on Mentally Abnormal Offenders. HMSO, 1975

13 Schizophrenia Commission. The Abandoned Illness: a Report by the Schizophrenia Commission. Rethink Mental Illness, 2012.

14 Higgins J. Four years' experience of an interim secure unit. Br Med J (Clin Res Ed) 1981; 14: 889-93.

15 Gudjonsson GH, MacKeith JA. A regional interim secure unit at the Bethlem Royal Hospital - the first fourteen months. Med Sci Law 1983; 23(3): 209-19.

16 Faulk M, Taylor JC. Psychiatric interim regional secure unit: seven years' experience. Med Sci Law 1986; 26(1): 17-22.

17 Dell S, Robertson G, Parker E. Detention in Broadmoor. Factors in length of stay. Br J Psychiatry 1987; 150: 824-7.

18 Tidmarsh D. Psychiatric risk, safety cultures and homicide inquiries. J Forensic Psychiatry Psychol 2008; 8: 138-51.

19 Burns T, Priebe S. Mental health care failure in England - myth and reality. Br J Psychiatry 1999; 174: 191-2.

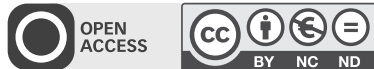

